\title{
Quantified pathology of emphysema, pneumoconiosis, and chronic bronchitis in coal workers
}

\author{
J LEIGH, K G OUTHRED, ${ }^{*} \mathrm{H}$ I MCKENZIE, ${ }^{*}$ M GLICK, AND A N WILES \\ From the Joint Coal Board, Medical Division, Sydney, New South Wales, Australia
}

ABSTRACT From a continuous series of 886 postmortem examinations on coal mine workers in New South Wales, Australia, from 1949 to 1982 and their histories the following data were obtained: age at death (886 cases), percentage of emphysema in both lungs (Heard method) (870 cases), bronchial gland wall (G-W) ratio (Reid Index) (412 cases), chest radiograph within 10 years of death (792 cases), history of work at the coal face (844 cases), history of amount of tobacco smoked (606 cases), and $\mathrm{FEV}_{1}$ five years before death (278 cases). Linear regression analysis showed the following: (1) The severity of emphysema had a significant positive regression on years of face work independently of age at death. (2) The severity of emphysema had a significant positive regression on the severity of $x$-ray pneumoconiosis, which was best defined in the non-smoking group and the non-bronchitic group. (3) There was a significant multiple linear regression relationship between severity of emphysema (dependent variable) and pneumoconiosis and G-W (independent variables). The ratio of standardised regression coefficients was pneumoconiosis: $\mathrm{G}-\mathrm{W}=3: 1$. (4) The severity of $x$-ray pneumoconiosis had a significant positive regression on years of face work and a negative regression on smoking amount. (5) G-W ratio had significant positive regressions on age of death and severity of emphysema but not years of face work or severity of pneumoconiosis. (6) Smoking was not correlated with severity of emphysema or G-W ratio. (7) $\mathrm{FEV}_{1}$ (\% predicted) was significantly negatively correlated with bronchitis, emphysema, and pneumoconiosis. (8) Severity of pneumoconiosis and emphysema have declined slightly but significantly over the 33 year period, but there has been no significant change in G-W ratio during 1960-82.

The analyses of postmortem data obtained from the lungs of 186 coal workers and associated clinical and physiological data from the same workers have allowed several conclusions to be drawn about pneumoconiosis, emphysema, and bronchitis in the coal industry. ${ }^{1}$ The main conclusions were:

(1) There is a quantitative relationship between the severity of emphysema postmortem and the postmortem severity of pneumoconiosis or $x$-ray pneumoconiosis in radiographs taken less than five years before death.

(2) Both postmortem emphysema and postmortem pneumoconiosis are quantitatively related to

${ }^{*}$ Dr Outhred and Dr McKenzie died during the preparation of
this paper.

Received 9 August 1982

Accepted 30 September 1982 exposure to coal dust as estimated by history of years worked at the face.

(3) Although there are correlations between the symptoms and signs of chronic bronchitis and history of coal dust exposure, there is no significant correlation between postmortem severity of chronic bronchitis (gland wall ratio $(\mathrm{G}-\mathrm{W})$ ) and exposure.

(4) There is a significant multiple regression relationship between postmortem emphysema (dependent variable) and postmortem pneumoconiosis and postmortem chronic bronchitis (independent variables). The ratio of standardised regression coefficients is pneumoconiosis: $\mathrm{G}-\mathrm{W}=1 \cdot 8: 1$.

(5) Neither postmortem emphysema nor postmortem chronic bronchitis are correlated with smoking history.

The previous study used a selected sample requiring complete data for all variables studied, including 
clinical data, and required a radiograph five years before death. No separate analysis by age groups was undertaken to separate age exposure effects and the postmortem pneumoconiosis scale used could only be claimed to be semi-quantitative.

The present study reports similar and more detailed analyses on a much larger sample of lungs from dead coal workers (886) with the aim of further clarifying the quantified aetiology and associations between pneumoconiosis, emphysema, and chronic bronchitis.

\section{Methods}

The sample included material from coal workers dying between 1949 and 1982 . The sampling fraction was about $22 \%$ of all deaths in miners. Pathology methods were generally as described in the previous study. ${ }^{1}$ Since 1972 , however, main bronchi have been used to assess gland wall ratio rather than the smaller bronchi used before that date (mean external diameter $5.4 \mathrm{~mm}$ ). In addition, since 1972 emphysema and G-W ratio measurements have been made by a different observer (JL).

To compare standards of assessment, this observer made quantitative estimates of emphysema by the Heard method on 400 lungs previously assessed by the former observer (KGO) and obtained good agreement, the mean difference being less than $5 \%$ emphysema, thus not affecting the $10 \%$ classification interval. All rereadings were done without knowledge of the former reading. To use more data, the criterion for inclusion of a case in the analyses related to pneumoconiosis was slackened to allow for a radiograph less than 10 years before death rather than five years as in the previous study. In over $90 \%$ of cases a radiograph taken within five years of death was available. Although the postmortem pneumoconiosis scale is a more valid estimate of pneumoconiosis in a logical sense and is highly correlated with the $x$-ray scale $(\mathrm{r}=0.78)$, the scale used in the previous study could be criticised for a subjective element, especially as it is impossible to examine a lung section for emphysema without noticing pneumoconiosis. To overcome this objection it was decided to use the ILO $x$-ray classification of pneumoconiosis on the most recent radiograph before death. The 1980 classification was used and a consensus classification of six observers was made with all earlier films being reread. Thirty-three cases with progressive massive fibrosis were included in the analysis.

The distribution of cause of death and the proporton of compensated to non-compensated workers in the sample remained essentially the same as in the previous study. ${ }^{1}$

\section{Variables analysed}

(1) Age at death (years).

(2) Years of face work.

(3) $X$-ray pneumoconiosis score ILO 1980

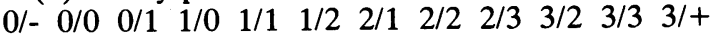

$\begin{array}{llllllllllll}1 & 2 & 3 & 4 & 5 & 6 & 7 & 8 & 9 & 10 & 11 & 12\end{array}$

(4) Emphysema score (Heard method) nil $=1$, $0-10 \%=2,10-20 \%=3,20-30 \%=4,30-40 \%=$ $5,40-50 \%=6$, and $>50 \%=7$.

(5) G-W ratio (Reid index).

(6) $\mathrm{Oz} / \mathrm{wk}$ tobacco smoked (lifetime average).

(7) $\mathrm{FEV}_{1}$ (\% predicted from Ferris et $\left.a l^{2}\right)$ (accepted only if $<$ five years before death).

\section{Data analysis}

The data were analysed using the BMDP 81 packages $6 \mathrm{D}$ and $1 \mathrm{R}$ on a Univac 1108 computer.

\section{Results}

Means and standard deviations of all variables analysed are shown in table 1. Frequency distributions of all variables analysed are shown in tables 2-8.

The relationships between emphysema, pneumoconiosis, and bronchitis with aetiological factors were studied by simple linear regression analysis on whole groups with complete data for the dependent and independent variables and on subgroups defined by categories of a third variable.

Inter-relationships between the three pathological criteria were studied by multiple regression analysis. $\mathrm{Bi}$-variate scattergrams were produced for all two variable relationships and in all cases visual inspection gave no justificaion for postulating anything other than a linear model.

Table 1 Means and standard deviations of variables analysed

\begin{tabular}{lccc}
\hline & Mean & $S D$ & No \\
\hline Age at death & 62.3 & $10 \cdot 9$ & 886 \\
Years of face work & 21.4 & $12 \cdot 6$ & 844 \\
$X$-ray pneumoconiosis score & $4 \cdot 6$ & $2 \cdot 3$ & 792 \\
Emphysema score & $3 \cdot 6$ & $1 \cdot 5$ & 870 \\
G-W ratio & 0.42 & $0 \cdot 14$ & 412 \\
Oz/wk tobacco smoked & $2 \cdot 9$ & $2 \cdot 0$ & 606 \\
FEV (\% predicted) & $72 \cdot 1$ & 27.1 & 278 \\
\hline
\end{tabular}

Table 2 Age at death (years)

\begin{tabular}{lllllllllll}
\hline$\leqslant 40$ & $41-50$ & $51-55$ & $56-60$ & $61-65$ & $66-70$ & $71-75$ & $>75$ & Total \\
\hline No 35 & 88 & 90 & 133 & 154 & 173 & 137 & 76 & 886 \\
$\%$ & $4 \cdot 0$ & $10 \cdot 0$ & $10 \cdot 0$ & $14 \cdot 8$ & $17 \cdot 2$ & $19 \cdot 4$ & $15 \cdot 4$ & $8 \cdot 5$ & \\
\hline
\end{tabular}


Table 3 Years of face work

\begin{tabular}{|c|c|c|c|c|c|c|c|c|c|c|c|}
\hline & $0-5$ & $6-10$ & $11-15$ & $16-20$ & $21-25$ & $26-30$ & $31-35$ & $36-40$ & $41-45$ & $>45$ & Total \\
\hline $\begin{array}{l}\text { No } \\
\%\end{array}$ & $\begin{array}{l}135 \\
16.0\end{array}$ & $\begin{array}{l}54 \\
6 \cdot 3\end{array}$ & $\begin{array}{l}68 \\
8 \cdot 0\end{array}$ & $\begin{array}{l}119 \\
14 \cdot 1\end{array}$ & $\begin{array}{l}109 \\
12.9\end{array}$ & $\begin{array}{l}147 \\
17.4\end{array}$ & $\begin{array}{l}119 \\
14 \cdot 1\end{array}$ & $\begin{array}{l}47 \\
5 \cdot 5\end{array}$ & $\begin{array}{l}37 \\
4 \cdot 4\end{array}$ & $\begin{array}{l}9 \\
1 \cdot 1\end{array}$ & 844 \\
\hline
\end{tabular}

Table $4 \quad X$-ray pneumoconiosis score

\begin{tabular}{lllllllllllll}
\hline & 1 & 2 & 3 & 4 & 5 & 6 & 7 & 8 & 9 & 10 & 11 & 12 \\
& $0 /$ & $0 / 0$ & $0 / 1$ & $1 / 0$ & $1 / 1$ & $1 / 2$ & $2 / 1$ & $2 / 2$ & $2 / 3$ & $3 / 2$ & $3 / 3$ & $3 /+$ \\
\hline $\begin{array}{l}\text { No } \\
\%\end{array}$ & 3 & 123 & 186 & 172 & 94 & 56 & 49 & 56 & 13 & 15 & 23 \\
\hline
\end{tabular}

Table 5 Emphysema score

\begin{tabular}{lcccccccc}
\hline & 1 & 2 & 3 & 4 & 5 & 6 & 7 & Total \\
\hline No & 47 & 191 & 235 & 169 & 122 & 56 & 51 & 870 \\
$\%$ & 5.4 & 21.9 & 27.0 & 19.3 & 14.0 & 6.4 & 5.9 & \\
\hline
\end{tabular}

\section{EMPHYSEMA}

Relationship with years at the coal face

Emphysema score was correlated significantly with years worked at the coal face $(r=0.38, p<0.001, n$ $=844)$. The regression equation was: emphysema score $=0.047$ years +2.57 .

To allow for the correlation between years at face and age, the regression of emphysema against years at face was calculated separately for different age groups. The results are shown in table 9 . The regression of emphysema score on years at the face is significant in the 56-60, 66-70, and over 75 age groups and the regression coefficients are higher in older groups suggesting an effect of both age and dust exposure.

Relationship with pneumoconiosis

Emphysema score was correlated significantly with $x$-ray pneumoconiosis $(\mathrm{r}=0.48, \mathrm{p}<0.001, \mathrm{n}=$ $777)$. The regression equation was: emphysema score $=0 \cdot 323$ pneumoconiosis $+2 \cdot 09$.

This relationship was similar in each age group, indicating that it was not simply an age effect (table 10).

The relationship between emphysema and pneumoconiosis was obtained for different smoking
Table 7 oz/wk tobacco

\begin{tabular}{lllccc}
\hline & 0 & $1-3$ & $4-6$ & $>6$ & Total \\
\hline No & 91 & 304 & 187 & 24 & 606 \\
$\%$ & 14.7 & $50 \cdot 1$ & $31 \cdot 2$ & 3.9 & \\
\hline
\end{tabular}

Table 8 FEV, (\%)

\begin{tabular}{llllll}
\hline & $\leqslant 30$ & $31-50$ & $51-80$ & $>80$ & Total \\
\hline No & 17 & 54 & 90 & 117 & 278 \\
$\%$ & $6 \cdot 1$ & $19 \cdot 4$ & $32 \cdot 4$ & $42 \cdot 1$ & \\
\hline
\end{tabular}

Table 9 Linear regression of emphysema score on years worked at face for different age groups

\begin{tabular}{lllll}
\hline Age at death & $b$ & $r$ & No & $p(<)$ \\
\hline$<40$ & $0 \cdot 014$ & $0 \cdot 12$ & 34 & NS \\
$41-50$ & $0 \cdot 008$ & $0 \cdot 08$ & 88 & NS \\
$51-55$ & $0 \cdot 014$ & $0 \cdot 21$ & 86 & NS \\
$56-60$ & $0 \cdot 008$ & $0 \cdot 15$ & 125 & $0 \cdot 05$ \\
$61-65$ & $0 \cdot 027$ & $0 \cdot 18$ & 145 & NS \\
$66-70$ & $0 \cdot 020$ & $0 \cdot 18$ & 164 & $0 \cdot 05$ \\
$71-75$ & $0 \cdot 023$ & $0 \cdot 16$ & 130 & NS \\
$>75$ & 0.025 & $0 \cdot 26$ & 72 & $0 \cdot 05$ \\
All & $0 \cdot 047$ & 0.38 & 844 & 0.001 \\
\hline
\end{tabular}

$\mathrm{b}=$ Regression coefficient .

$r=$ Correlation coefficient

groups: non, light ( $\leqslant 3 \mathrm{oz} / \mathrm{wk})$, medium $(3-6 \mathrm{oz} /$ wk), heavy ( $>6 \mathrm{oz} / \mathrm{wk})$. The results are shown in table 11 from which it may be seen that the relationship is strongest and the regression coefficient highest in non-smokers.

To eliminate the effect of correlation between

Table $6 \quad G-W$ ratio

\begin{tabular}{lllllllll}
\hline & $0 \dashv-0.2$ & $0.21-0 \cdot 3$ & $0.31-0.4$ & $0.41-0.5$ & $0.51-0 \cdot 6$ & $0.61-0.7$ & $0.71-0 \cdot 8$ & Total \\
\hline No & 14 & 53 & 154 & 99 & 53 & 24 & 15 & 412 \\
$\%$ & 3.4 & 12.9 & 37.4 & $24 \cdot 0$ & 12.9 & $5 \cdot 8$ & 3.6 & \\
\hline
\end{tabular}


Table 10 Linear regression of emphysema score on $x$-ray pneumoconiosis score for different age groups

\begin{tabular}{|c|c|c|c|c|}
\hline Age at death & $b$ & $r$ & No & $p(<)$ \\
\hline $\begin{array}{l}<40 \\
41-50 \\
51-55 \\
56-60 \\
61-65 \\
66-70 \\
71-75 \\
>75 \\
\text { All }\end{array}$ & $\begin{array}{l}0.66 \\
0.26 \\
0.29 \\
0.31 \\
0.22 \\
0.24 \\
0.19 \\
0.17 \\
0.32\end{array}$ & $\begin{array}{l}0.66 \\
0.35 \\
0.32 \\
0.40 \\
0.29 \\
(0.41 \\
0.31 \\
0.34 \\
0.48\end{array}$ & $\begin{array}{r}31 \\
81 \\
84 \\
121 \\
133 \\
154 \\
111 \\
62 \\
777\end{array}$ & $\begin{array}{l}0 \cdot(001 \\
0 \cdot(01 \\
0 \cdot(01 \\
0 \cdot(0) 1 \\
0 \cdot(001 \\
0 \cdot(001 \\
0 \cdot(001 \\
0 \cdot(01 \\
0 \cdot 001\end{array}$ \\
\hline
\end{tabular}

$\mathrm{b}=$ Regression coefficient.

$r=$ Correlation coefficient.

emphysema and chronic bronchitis on the relationship between emphysema and pneumoconiosis, two approaches were used.

(a) The multiple linear regression of emphysema on pneumoconiosis and $\mathrm{G}-\mathrm{W}$ was calculated. The multiple regression equation was:

emphysema $=0.27$ pneumoconiosis $+1.41 \mathrm{G}-\mathrm{W}+$

$$
\begin{aligned}
& (\mathrm{t}=8 \cdot 29) \\
& \text { multiple } \mathrm{R}=0.41^{1.65}
\end{aligned}(\mathrm{t}=2.78)^{1.65}
$$

The standardised regression coefficients were: pneumoconiosis 0.38
G-W
$0 \cdot 12$

The simple correlation between pneumoconiosis and G-W was $0 \cdot 02$ (non-significant).

(b) The linear regression of emphysema on pneumoconiosis was calculated for different chronic bronchitis groups (normal G-W $\leqslant 0.3$, mild $0.3<$ $\mathrm{G}-\mathrm{W} \leqslant 0.55$, severe $\mathrm{G}-\mathrm{W}>0.55)$.

The results are shown in table 12 .

Table 11 Linear regression of emphysema score on $x$-ray pneumoconiosis score for different smoking habits

\begin{tabular}{lllll}
\hline Smoking amount & $b$ & $r$ & No & $p(<)$ \\
\hline Nil & 0.49 & $(0.76$ & 91 & $(0 \cdot()(01$ \\
Light & 0.24 & 0.34 & 288 & $(0 \cdot 01$ \\
Medium & 0.30 & 0.39 & 181 & $0 \cdot()(01$ \\
Heavy & 0.47 & $(0.34$ & 23 & NS \\
All & $(0.32$ & 0.48 & 777 & $(0 \cdot()(01$ \\
\hline
\end{tabular}

$\mathrm{b}=$ Regression coefficient.

$r=$ Correlation coefficient

Table 12 Linear regression of emphysema on pneumoconiosis in different bronchitis groups

\begin{tabular}{lllrl}
\hline Bronchitis & $b$ & $r$ & No & $p(<)$ \\
\hline Nil & $(0.37$ & 0.43 & 82 & 0.001 \\
Mild & 0.26 & 0.40 & 241 & 0.001 \\
Severe & 0.19 & 0.26 & 70 & 0.05 \\
\hline
\end{tabular}

$\mathrm{b}=$ Regression coefficient.

$r=$ Correlation coefficient.
The relationship becomes stronger and the slope of the regression line greater with lesser degrees of bronchitis.

The overall correlation of emphysema score with G-W ratio was $0.13(\mathrm{p}<0.005, \mathrm{n}=404)$. The simple regressions of emphysema score on smoking, age of death, FEV1\%, and chronological time of death are shown in table 13. There is a significant positive regression on age of death and a significant negative regression on FEV1\%.

The negative regression on chronological time (arbitrary units) indicates that the prevalence of emphysema in the industry has been reducing over the 33 years in which necropsies have been performed. Nevertheless, the effect is slight $(0.02$ of an emphysema category or $0.2 \%$ lung emphysema per year) and may safely be ignored in the interpretation of the results. No relationship between emphysema and smoking was found $(\mathrm{r}=0.01$, non-significant, $\mathrm{n}$ = 594).

\section{PNEUMOCONIOSIS}

Relationship with years at coal face

Table 14 shows the linear regression of $x$-ray pneumoconiosis on years of face work for different age groups. In every age group except the two of smallest size (the oldest and youngest) there is a significant relationship between history of years at

Table 13 Linear regression of emphysema score on various independent variables

\begin{tabular}{lccll}
\hline & $b$ & $r$ & No & $p(<)$ \\
\hline Age at death & 0.063 & 0.44 & 870 & $0 \cdot 001$ \\
$\begin{array}{l}\text { Chronological time } \\
\text { of death }\end{array}$ & -0.0007 & -0.12 & 870 & $0 \cdot 001$ \\
FEV1\% & -0.030 & -0.51 & 272 & $0 \cdot 001$ \\
Smoking amount & 0.008 & 0.01 & 594 & NS \\
\hline
\end{tabular}

$\mathrm{b}=$ Regression coefficient.

$r=$ Correlation coefficient.

Table 14 Linear regression of $x$-ray pneumoconiosis severity on years of face work for different age groups

\begin{tabular}{llrrl}
\hline Age at death & $b$ & $r$ & No & $p(<)$ \\
\hline$<40$ & $0 \cdot() 0$ & $-0 \cdot 02$ & 31 & NS \\
$41-50$ & $0 \cdot 037$ & $0 \cdot 30$ & 83 & $0 \cdot(1)$ \\
$51-55$ & $0 \cdot 056$ & $0 \cdot 41$ & 86 & $0 \cdot 001$ \\
$56-60$ & $0 \cdot 051$ & $0 \cdot 30$ & 124 & $0 \cdot 001$ \\
$61-65$ & $0 \cdot 057$ & $0 \cdot 32$ & 134 & $0 \cdot 001$ \\
$66-70$ & $0 \cdot 063$ & $0 \cdot 26$ & 154 & $0 \cdot 01$ \\
$71-75$ & $0 \cdot 047$ & $0 \cdot 21$ & 112 & $0 \cdot 05$ \\
$>75$ & $0 \cdot 055$ & $0 \cdot 24$ & 64 & NS \\
All & $0 \cdot 084$ & 0.45 & 788 & $0 \cdot(0) 1$ \\
\hline
\end{tabular}

$\mathrm{b}=$ Regression coefficient .

$r=$ Correlation coefficient . 
Table 15 Linear regression of $x$-ray pneumoconiosis severity on years of face work for different smoking amounts

\begin{tabular}{lllrl}
\hline Smoking amount & $b$ & $r$ & No & $p(<)$ \\
\hline Nil & 0.099 & 0.55 & 93 & 0.001 \\
Light & 0.065 & 0.37 & 293 & 0.001 \\
Medium & 0.056 & 0.33 & 184 & 0.001 \\
Heavy & 0.069 & 0.61 & 24 & 0.01 \\
\hline
\end{tabular}

$\mathrm{b}=$ Regression coefficient

$r=$ Correlation coefficient.

face work and $x$-ray pneumoconiosis. The overall regression equation is:

pneumoconiosis $=0.084$ years at face $+2.82(\mathrm{r}=$ $0.45, \mathrm{p}<0.001, \mathrm{n}=788$ ).

The relationship with face work was also calculated separately in different smoking groups; these results are shown in table 15 . The regression with face work is greatest in the non-smoking group, next in the heavy smoking group, and least in the intermediate smoking groups. The mean values of pneumoconiosis score in the four smoking groups are: nil $: 4 \cdot 25$, light $: 4 \cdot 46$, medium $: 3 \cdot 88$, heavy : $3 \cdot 25$.

\section{Relationship with smoking}

Overall the regression of pneumoconiosis on smoking was: pneumoconiosis $=-0.13(\mathrm{oz} / \mathrm{wk})+4.57(\mathrm{r}$ $=-0.12, \mathrm{p}<0.005, \mathrm{n}=595)$.

\section{Relationship with age}

The regression relationship was: pneumoconiosis $=$ 0.102 age $-1.76(\mathrm{r}=0.47, \mathrm{p}<0.001, \mathrm{n}=792)$.

\section{Relationship with chronological time}

There was a significant decline $(\mathrm{p}<0.01)$ in the severity of $x^{2}$ ray pneumoconiosis at the last radiograph over the 33 years of the study. This corresponded to a fall of $1 / 12$ ILO category in 33 years and may be safely ignored in interpretation of results.

\section{Relationship with FEV1\%}

There was a significant negative correlation with FEV1\% $(r=-0.24, p<0.001, n=278)$, explicable in terms of the emphysema associated with pneumoconiosis.

\section{BRONCHITIS}

Severity of postmortem chronic bronchitis, as measured by G-W ratio, has no significant correlation with years of face work, smoking history, severity of pneumoconiosis, or chronological time.

G-W ratio was significantly correlated with age at death $(r=0.13, p<0.005, n=411)$, emphysema score $(r=0.13, p<0.005, n=401$,$) and FEV1\%$ $(\mathrm{r}=-0 \cdot 15, \mathrm{n}=236, \mathrm{p}<0.05)$.
SMOKING

In addition to the effects described above, there was an overall weak negative correlation between age of death and smoking history $(\mathrm{r}=-0.08, \mathrm{p}<0.05, \mathrm{n}$ $=606$ ).

\section{Discussion}

The results confirm and clarify those of our previous study and much of the discussion reported therein still applies. ${ }^{1}$ In addition, however, we have shown that postmortem emphysema is related to dust exposure independently of age. The relationship between emphysema and pneumoconiosis has been shown more clearly and the effects of smoking distinguished. It is striking that the regression coefficient of emphysema on pneumoconiosis is highest in the non-smoking group.

By considering the relationship between emphysema and pneumoconiosis in non-bronchitic subjects any "blurring" effects of centrilobular and panacinar emphysema included in the total emphysema estimate are minimised, and the relationship between emphysema (almost exclusively focal) and pneumoconiosis is still more clearly seen.

The negative correlation found between smoking amount and severity of pneumoconiosis may be explained in several different ways. Firstly, lighter smokers live longer and thus have more time to accumulate dust and hence get more pneumoconiosis. Secondly, although no correlation between smoking and gland wall ratio was shown, there is a correlation between clinical chronic bronchitis and smoking, and other workers have suggested that increased sputum secretion may prevent alveolar deposition of particles. ${ }^{3}$ Thirdly, and possibly most likely, there is a negative correlation between face work and amount smoked ${ }^{1}$ (for the obvious reason that men cannot smoke underground at all or at least never at the face) and hence smokers work least at the face and get less dust exposure. Fourthly, the postmortem selection process ${ }^{1}$ selects those who have relatively more chance of having pneumoconiosis and relatively less chance of being heavy smokers - that is, are more likely to be compensated.

The findings in relation to G-W ratio and FEV1\% confirm the previous work, and suggest that studies on industrial bronchitis are best carried out on the living work force population.

The inclusion of cases with progressive massive fibrosis is justified by the fact that the ILO $x$-ray classification is of the background nodularity and that emphysema estimations will, if anything, be artifically reduced by space occupying PMF lesions and hence the conclusions represent an underesti- 
mate of the relationship between emphysema and pneumoconiosis or exposure.

As before, all forms of emphysema were included in the quantification and, although most emphysema was focal in type, the inclusion of centrilobular (non-focal) and panacinar emphysema is reflected in the variance of the calculated statistics. Nearly all PMF cases were, in the earlier part of the series, dying before $F E V_{1}$ measurements were routinely made, so the explanation for the significant negative correlation of pneumoconiosis $x$-ray grade with FEV1\% (not found in the previous study with a smaller number of cases) is not an independent effect of PMF as found by Lyons and Campbell ${ }^{4}$ and is presumably due to emphysema alone.

It is interesting to note that the overall multiple regression relationship between emphysema, pneumoconiosis, and chronic bronchitis in the larger data set gives a ratio of standardised regression coefficients: pneumoconiosis:chronic bronchitis = $3: 1$ compared to $1 \cdot 8: 1$ in the earlier study, reinforcing further the hypothesis that emphysema in coal workers is primarily related to coal dust.
We thank Dr K White for expert help with pulmonary pathology and $\mathrm{Dr}$ I Southgate, director of the Joint Coal Board Pathology Laboratory, and Mr D Moffat for skilled technical help.

\section{References}

' Leigh J, Outhred KG, McKenzie HI, Wiles AN. In: Walton WH, ed. Multiple regression analysis of quantified aetiological, clinical and postmortem pathological variables related to respiratory disease in coal workers. Inhaled particles $V$. Oxford: Pergamon Press, 1982. Ann Occup Hyg 1982;26:383-400.

${ }^{2}$ Ferris BG, Anderson DO, Zickmantel R. Prediction values for screening tests of pulmonary function. Am Rev Resp Dis 1965;91:252-61.

${ }^{3}$ Jacobsen M, Burns J, Attfield MD. Smoking and coal workers' simple pneumoconiosis. In: Walton WH, ed. Inhaled particles IV. Oxford: Pergamon Press, 1977:759-72.

- Lyons JP, Campbell H. Relation between progressive massive fibrosis, emphysema, and pulmonary dysfunction in coal workers' pneumoconiosis. Br J Ind Med 1981;38:125-9. 